\title{
Meilensteine im Erwerb der deutschen Orthografie
}

\author{
Irene Corvacho del Toro', Günther Thomé2
}

${ }^{1}$ Germanistisches Seminar, Universität Siegen

2Institut für sprachliche Bildung GbR, Oldenburg

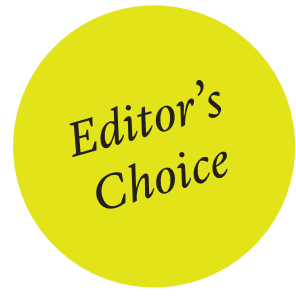

\begin{abstract}
Zusammenfassung: Hintergrund: Noch immer stellt eine Modellierung der deutschen Orthografie, die sprachtheoretisch fundiert und rechtschreibdidaktisch anwendbar ist, ein Forschungsdesiderat dar. Diese Arbeit möchte einen Beitrag hierzu leisten und ein fachdidaktisch orientiertes Modell zu Meilensteinen des Orthografieerwerbs vorstellen. Methode: Entlang der Sprachebenen, die die deutsche Rechtschreibung abbildet, wird gezeigt, welche Meilensteine Lernende bis zur vollentfalteten Rechtschreibkompetenz durchlaufen. Grundlage dieser Analyse bilden die Rechtschreibnorm sowie Rechtschreibfehler aus Interventionsstudien. Ergebnisse: Das Modell verdeutlicht die zentrale Stellung der Einheiten Wort und Morphem im deutschen Schriftsystem und für den Rechtschreiberwerb. Darüber hinaus werden Meilensteine, die mit phonologischen und morphosyntaktischen Fähigkeiten zusammenhängen, von solchen, die auf die morphologische Bewusstheit rekurrieren, unterschieden. Diskussion: Die Modellierung legt nahe, nach der Einführung von Basisgraphemen die prototypischen Wortstrukturen und den Wortaufbau des Deutschen ins Zentrum der Rechtschreibdidaktik zu stellen. Persistierenden Rechtschreibfehler werden in Abhängigkeit von der Stärke ihres Zusammenhangs entweder mit Spracherwerbs- oder Rechtschreiberwerbsprozessen erklärt. Das Modell kann in der Diagnostik, der Lehrerbildung, der Rechtschreibdidaktik und der Modellierung Rechtschreibkompetenz Anwendung finden.
\end{abstract}

Schlüsselwörter: Deutsche Orthografie, phonologische und morphologische Bewusstheit, Rechtschreibfehler, Rechtschreibdidaktik, Schriftspracherwerb und Rechtschreiberwerb

\section{Milestones in the Acquisition of German Orthography}

Abstract: Introduction: A model of German orthography that is well-founded in terms of language theory and can be used in terms of spelling didactics is still a research desideratum. This work aims to make a contribution and presents a didactically-oriented model of milestones in the acquisition of German orthography. Method: Along the language areas that the German spelling depicts, it is shown which milestones learners pass through to fully developed spelling skills. This analysis is based on the spelling norm as well as spelling mistakes gathered from intervention studies. Results: The model illustrates the central role of the linguistic units word and morpheme in the German writing system and for its acquisition. In addition, milestones related to phonological or morphosyntactic skills are distinguished from those related to morphological awareness. Discussion: This modeling suggests that after the introduction of basic grapheme correspondences (Basisgrapheme) the prototypical word structures and word composition should be at the center of spelling didactics. Persistent spelling errors are explained depending on the strength of their connection with either language or spelling acquisition processes. The model can be applied in diagnostics, teacher training, spelling didactics and the modeling of spelling competence.

Keywords: German orthography, phonological and morphological awareness, didactics of orthography, spelling mistakes, spelling acquisition

\section{Einleitung}

Die Modellierung der deutschen Orthografie schaut auf eine lange Tradition von theoretischen Arbeiten zurück. Verschiedene orthografische Prinzipien, die die Schreibnorm begründen, wurden in verschiedenen Variationen in Anzahl und Gehalt postuliert (vgl. Nerius, 2007; Munske, 2005; Eisenberg, 2017; Thomé, 2019). Diese Arbeiten nahmen stets sowohl eine linguistische als auch eine fach- didaktische Perspektive ein (vgl. ebd.). Spannungsfelder bestanden in der Abgrenzung zwischen deskriptivem (beschreibenden) und präskriptivem (vorschreibenden) Vorgehen, aber auch zwischen einer diachronen (historischen) und / oder synchronen (gegenwärtigen) Beschreibung (vgl. Nerius, 2007, S. 96-97).

Thomé (1992, zuletzt 2019) erarbeitet angelehnt an Frequenz und der Markiertheitstheorie von Jakobson (1969; vgl. auch Trubetzkoy, 1989) eine statistisch deskriptive 
Modellierung der Orthografie. Unter Anwendung einer computerbasierten Auszählung wurden aus einem Märchen- und Romankorpus in einem ersten Schritt für jedes deutsche Phonem das Graphem ermittelt, welches es repräsentiert. In einem zweiten Schritt wurde für jede festgestellte Phonem-Graphem-Korrespondenz das Graphem ermittelt, das am häufigsten auftritt. Diese Phonem-Graphem-Korrespondenz wurden in Anlehnung an Augst (1974) Basisgraphem genannt.

Die Ergebnisse dieser Analysen zeigten, dass 100\% der Phoneme in den deutschen Texten durch je ein Graphem wiedergegeben werden. Die phonematische Basis des deutschen Schriftsystems, also sein alphabetischer Schrifttyp, wurde somit belegt und eine Definition des Graphems in Abhängigkeit vom Phonem empirisch begründet. Darüber hinaus zeigte diese statistische Auszählung, dass in einem üblichen deutschen Text (hier Kindermärchen und Romantexte) $90 \%$ der Phoneme mit dem Basisgraphem wiedergegeben werden (vgl. letzte Auszählung in Thomé, Siekmann \& Thomé, 2011; Thomé \& Thomé, 2016). Diese Basisgrapheme gelten der Markiertheitstheorie nach als die unmarkierten Phonem-Graphem-Korrespondenzen, denn sie kommen häufiger vor und enthalten über die Lautung hinaus keine weiteren Informationen.

Neben den Basiskorrespondenzen (90\%) umfasst der Bereich der orthographischen Markierungen, d.h. der weniger frequenten Phonem-Graphem-Korrespondenzen, die über die lautliche weitere Information enthalten (bspw. morphologische < Hund $>$ ), die restlichen 10\% der Korrespondenzen in einem üblichen deutschen Text. Diese Korrespondenzen werden Orthographeme genannt (Thomé, 1992). Hinter ihnen verbergen sich die orthographischen Phänomene, d.h. verschiedene Regelungen und Einschränkungen bei der Auswahl des zu schreibenden Graphems (vgl. Thomé, 1992; Corvacho del Toro, 2017).

Für die Systematisierung der Orthographeme, sodass Aussagen über die Auftretensbedingungen gemacht werden können, reicht die Analyse in Einzellauten nicht aus. Vielmehr müssen Orthographeme auf Wortebene analysiert werden, damit ihr Auftreten beschrieben werden kann. Analysen der Frequenz und Markiertheit ergeben, dass es Orthographeme gibt, die sich aus bestimmten Regelhaftigkeiten systematisch konstruieren lassen, und andere, die dies nicht erlauben (vgl. Corvacho del Toro, 2017). Demnach können Wortschreibungen mit Orthographemen in regelhafte und nicht regelhafte unterteilt werden. Wörter, die nicht regelhaft sind, sind in der Anzahl reduzierter, und die enthaltenen Phonem-Orthographem-Korrespondenzen lassen sich weder regelhaft konstruieren noch ableiten (bspw. <Vater, Chor >). Sie können dennoch aus dem Kernbereich des deutschen
Wortschatzes stammen und in der Nutzung frequent sein (bspw. <sehr, und >). Andere Wörter, die Orthographeme enthalten, entsprechen entweder Lehn- oder Fremdwortorthografien (bspw. <Bibel, Baby, Rhythmus $>$ ) und / oder ihre Schreibung ist historisch begründet (bspw. <Theater $>$, usus scribendi, Altorthographie).

Die satzinterne Großschreibung als Eigenheit der deutschen Orthografie ist ein Phänomen, das über der Ebene der Basis- und Orthographeme liegt (vgl. ${ }^{*}<$ vater $>$, $<$ Vater $>$; Anmerkung: Asterix steht vor ungrammatischen Wortformen.). Die linguistische Einheit zur Markierung ist die Majuskel $(<\mathrm{V}, \mathrm{N}, \mathrm{K}>\mathrm{etc}$.). Die Großschreibung lässt sich am systematischsten über die Phrasen- und/oder Satzstruktur beschreiben (Röber-Siekmeyer, 1999; Bredel, 2006, 2010; Granzow-Emden, 2013; Funke, 2017), da letztendlich die Position im Satzkontext die Wortart bestimmt. Die Satzzeichen sind ein Phänomen der Textstruktur, denn erst die Beachtung der Satzkonstruktion (Prädikat und Satzglieder) sowie das Verhältnis der Sätze zueinander erlaubt es, Satzgrenzen zu bestimmen und entsprechend Satzzeichen zu setzen. Diese vier sprachlichen Ebenen können als grundlegende strukturgebende Gestalt der deutschen Orthografie angenommen werden: Lautstruktur, Wortstruktur, Phrasen-/ Satzstruktur sowie Textstruktur (vgl. Nerius, 2007).

Zum Erreichen einer normkonformen Schreibung müssen diese sprachlichen Ebenen beachtet werden. Rechtschreibfehler wiederum liefern konkrete Hinweise darüber, welche dieser Ebenen nicht beachtet wurde und zum Fehler geführt hat (Corvacho del Toro, 2016b). Alle Modelle, die den Schriftspracherwerb beschreiben, sind dementsprechend Fehlermodelle (vgl. Siekmann \& Thomé, 2018; Scheerer-Neumann, 2014; Thomé \& Thomé, 2020).

\section{Forschungsziel}

Ausgehend von dieser theoretischen Darlegung (Basisund Orthographeme, regelhafte und nicht regelhafte markierte Schreibungen (Orthographeme) sowie Phänomene der Satz- und der Textebene) verfolgt dieser Beitrag das Ziel, eine idealisierte Lernprogression zu erarbeiten, die praktische Relevanz hat. Der Orthografieerwerb soll in Form von Meilensteinen beschrieben werden, um eine Orientierung für Diagnose, Unterricht, Forschung und Förderung anzubieten.

Folgende Prämissen liegen dieser Erarbeitung zugrunde:

1. Die deutsche Orthografie lässt sich entlang der linguistischen Sprachebenen, die sie abbildet, modellieren.

2. Das Erlernen der Orthografie kann über die qualitativquantifizierende Analyse von Rechtschreibfehlern beschrieben werden. 


\section{Methode}

\section{Forschungsrahmen}

Die vorliegende theoretische Arbeit bildete die Grundlage zur Erarbeitung von Unterrichtsmaterialien zur Förderung von Rechtschreibkompetenzen im Rahmen einer Lehrerfortbildungs- und Interventionsstudie (Schuljahr 2019 / 20). Das ursprüngliche Forschungsdesign sah Lehrerfortbildungen und eine Unterrichtsintervention über 20 Wochen vor. Die Stichprobe umfasste 480 Gymnasialschüler_innen der 5. und 6. Klassenstufen in 16 Frankfurter Schulklassen. Zehn Lehrkräfte wurden im Rahmen von Lehrerfortbildungen und durch Begleitung des wöchentlichen stattfindenden Rechtschreibunterrichts qualifiziert. Zusätzlich kamen die für die Interventionsstudie entwickelten Unterrichtsmaterialien zur Anwendung. Dieses Coaching-Angebot hatte zum Ziel, handlungsnah die Umsetzung des rechtschreibdidaktischen Konzepts zu unterstützen, sodass das Erlernte sicher und nachhaltig verankert werden konnte. Für die Überprüfung der Wirksamkeit dieser Intervention wurde ein Experimental-/Kontrollgruppendesign mit 10 Klassen in der Experimentalgruppe, 4 Klassen in einer Kontrollgruppe mit alternativer Zuwendung (Training im Bereich Wortschatz) und 2 weiteren Kontrollklassen ohne Zuwendung erarbeitet. Leistungsmaße umfassten die standardisierte Rechtschreibleistung, ein selbstkonstruiertes Diktat, ein selbstkonstruiertes Instrument zur Erhebung des Bildungswortschatzes sowie Unterrichtsbeobachtungen und die Befragung der teilnehmenden Lehrkräfte. Die Studie startete im September 2019 mit der Eingangserhebung, danach folgten die Fortbildungen und schließlich begann nach den Herbstferien 2019 die Unterrichtsbegleitung durch geschulte studentische Mitarbeiter_innen. Leider kam es durch die Corona-Pandemie im März 2020 zum Abbruch der Studie. Erhebungen an Schulen waren durch die Hygiene-Auflagen unerwünscht. Zudem wäre die Wirksamkeit der Intervention nach mehrwöchiger Unterbrechung und anschließender Teilung der Klassen, was eine Reduzierung der Unterrichtszeit um die Hälfte bedeutete, nicht mehr überprüfbar. Dieser Beitrag beschränkt sich demnach auf das theoretische Modell, welches die Grundlage für den rechtschreibdidaktischen Ansatz und die entwickelten Rechtschreibmaterialien darstellt (vgl. Corvacho del Toro, 2019).

\section{Annahmen und Modellierungskriterien}

Ausgangspunkt der theoretischen Modellierung sind die grundlegenden strukturgebenden Ebenen der Gestalt der Orthografie: Lautstruktur, Wortstruktur, Phrasen-/Satz- struktur sowie Textstruktur. Innerhalb dieser Ebenen werden die linguistischen Disziplinen verortet, die zur Beschreibung des Schriftsystems und des Rechtschreiberwerbs grundlegend sind: (0) Phonetik und (1) Phonologie, (2) Morphophonologie, (3) Morphologie, (4) Morphosyntax, (5) Syntax und (6) Lexik.

Folgende linguistischen Kriterien sind leitend für die Modellierung:

1. Die Unterscheidung zwischen Kern und Peripherie des Wortschatzes des Deutschen (vgl. Nerius, 2007, S.83 ff.).

2. Die Unterscheidung zwischen häufigen und selten vorkommenden linguistischen Einheiten (Basis-/Orthographeme, prototypische deutsche Wörter, Morpheme, Satzstrukturen) (vgl. Thomé, 1992; Eisenberg \& Fuhrhop, 2007; Elsen, 2014; Pittner \& Bermann, 2015).

3. Die Unterscheidung zwischen regelhaft und nicht regelhaft konstruierbarer Struktur, im Hinblick auf Generalisierbarkeit (vgl. Corvacho del Toro, 2016a; HoffmannErz, 2015).

4. Die Unterscheidung zwischen ableitbaren und nicht ableitbaren Schreibungen, im Hinblick auf rekurrierendes regelhaftes Muster oder Merkleistung (vgl. Corvacho del Toro, 2016a, 2017).

Bei der Erarbeitung dieser idealisierten Lernprogression werden psycholinguistische Prozesse als entscheidende Einflussfaktoren betrachtet und berücksichtigt. Rückschlüsse über die Sprachverarbeitung werden über Rechtschreibfehler begründet. In Bezug auf die psycholinguistischen Prozesse beruht diese Modellierung auf der Einteilung in systematische und unsystematische Rechtschreibfehler (vgl. Thomé \& Thomé, 2020; Thomé, Corvacho del Toro, Thomé, 2011) und auf folgende Annahmen über ihre Ursachen und ihrer Persistenz:

1. Systematische Rechtschreibfehler sind systembedingt und ergeben sich:

a. bei Überlappungen zwischen den verschiedenen linguistischen Disziplinen, die die Rechtschreibung berücksichtigt (bspw. phonetisch-phonologisch ${ }^{*}<$ seltn $>$, $<$ selten>),

b. bei Brüchen in der Regelhaftigkeit. Das betrifft Schreibungen, die als Merkschreibung bzw. Merkstrukturen eingeprägt werden müssen (bspw. durch Lehnwörter ${ }^{*}<$ Fiebel $>$, $<$ Fibel $>$ ).

2. Unsystematische (Rechtschreib)fehler ergeben sich durch: a. Schwierigkeiten in der Wahrnehmung von Phonemen (phonologische Beeinträchtigung bzw. phonologischer Förderbedarf, ${ }^{*}<$ Bost $>$ für $<$ Post $>$ ),

b. Schwierigkeiten in der Rektion und Kongruenz des Deutschen (grammatische Beeinträchtigung bzw. grammatischer Förderbedarf, *<schöne Auto>, $<$ schönes Auto>), 
c. fehlende Einsicht in die Systematik der deutschen Orthografie (morphematische und syntaktische Beeinträchtigung; bzw. morphematischer Förderbedarf, $\quad{ }^{*}<$ beckommen $>, \quad<$ bekommen $>$; ${ }^{*}<$ keinen $\operatorname{sinn}>,<$ keinen Sinn $>$ ).

3. Im Hinblick auf die Entwicklungsprognose wird Folgendes angenommen:

a. Persistierende (Rechtschreib)fehler haben ihren Ursprung entweder in einer phonologischen und/oder grammatischen Beeinträchtigung (siehe 2a und 2b).

b. Rechtschreibfehler, die eine fehlende Einsicht in die Systematik der deutschen Orthografie erkennen lassen (siehe 2c), haben ihren Ursprung wahrscheinlich in einer Beeinträchtigung des impliziten Lernens (vgl. Ise \& Schulte-Körne, 2012). Sie haben bei geeigneter Förderung eine gute Entwicklungsprognose (vgl. Corvacho del Toro, 2016b).

Implizites Lernen wird hierbei als die Fähigkeit, unbewusst und unbeabsichtigt Regelhaftigkeit bzw. statistische Zusammenhänge (d.h. Muster) aus der geschriebenen Sprache zu abstrahieren und auf neue Wörter anzuwenden, definiert. Ist das implizite Lernen beeinträchtigt, sind die Schüler_innen demnach umso abhängiger von einem gut strukturierten Vorgehen, das dieses unbewusste Ableiten von Regelhaftigkeit (d.h. das Mustererkennen) unterstützt. Dabei geht es nicht ausschließlich um visuelle, vielmehr geht es um komplexe sprachliche Muster, die sprachliche und schriftliche Ebenen berücksichtigt. Der Rechtschreibunterricht bzw. die Rechtschreibförderung muss demnach die Systematik der Orthografie klar veranschaulichen, direkte Instruktion einschließen und genügend Übungsgelegenheiten bieten. Das Sammeln von vielen strukturähnlichen Wörtern, die eine Regelhaftigkeit begründen, stellt u. a. ein solches Vorgehen dar.

Die oben vorgestellten Annahmen lassen schlussfolgern, dass phonologische oder grammatische Beeinträchtigungen (siehe 2a, 2b) eine systematische, kontinuierliche und längerfristige Förderung sowie die Entwicklung kompensatorischer Strategien zur Erreichung einer vollentfalteten Rechtschreibkompetenz bzw. grammatische Kompetenz erfordern. Dagegen ist bei Rechtschreibfehlern, die eine fehlende Einsicht in die Systematik der deutschen Orthografie erkennen lassen (siehe 2c), anzunehmen, dass diese über die Förderung der morphematischen Bewusstheit überwunden werden können (vgl. Galuschka et al., 2020; Görgen, De Simone, Schulte-Körne \& Moll, 2021; Volkmer, Schulte-Körne \& Galuschka, 2019; Kirby et al., 2012). Dieser Zusammenhang ist damit zu begründen, dass die Morphologie im Gegensatz zu phonologisch und/oder morphosyntaktisch geprägten Bereichen (Phonem- und Deklinationssystem) der Rechtschreibnorm im größeren Maße unabhängig vom frü- hen Spracherwerb durch sprachanalytische und schriftsystematische Operationen erlernt werden kann (siehe Abbildung 1). Dementsprechend liegt es nahe, anzunehmen, dass die Förderung der morphematischen Bewusstheit (vgl. Clahsen \& Fleischhauer, 2014) sich auf eine phonologische und/oder grammatische Beeinträchtigung kompensatorisch auswirken kann (vgl. Corvacho del Toro, 2016b; Kargl \& Landerl, 2018; Kargl, Wendtner, Purgstaller \& Fink, 2018).

Abbildung 1 stellt das Modell des Orthografieerwerbs vor. Die Darstellung soll veranschaulichen, dass die Rechtschreibleistung sowohl von phonetischen, phonologischen und morphosyntaktischen Kompetenzen, die im frühen Spracherwerb entwickelt wurden (vgl. Berg, 2005; Dahmen, 2012; Siekmann \& Corvacho del Toro, 2018), als auch von graphotaktischen, morphophonologischen, morphematischen und syntaktischen Kompetenzen, die stärker von angebotenem Lerninput und dem impliziten Lernen abhängig sind, beeinflusst wird. Morphophonologische Fähigkeiten, d.h. das Wahrnehmen von Kurz- und Langvokalen, die mit Orthographemen in der Schreibung markiert werden, stellen eine Besonderheit dar. Sie verzahnen beide Erwerbsverläufe eng miteinander, den Sprach- und den Orthografieerwerb (siehe Abb. 1, zweifarbiger Schriftverlauf).

\section{Ergebnisse}

\section{Meilensteine des Orthografieerwerbs}

Die Beschreibung der Meilensteine des Orthografieerwerbs berücksichtigt zunächst die grundlegenden Ebenen: Laut-, Wort-, Phrase-/Satz- und Textstruktur (siehe Tab. 1). Innerhalb von diesen Oberkategorien werden die linguistischen Disziplinen und die psycholinguistischen Prozesse beschrieben, die Schüler_innen durchlaufen bzw. die Einsichten, die sie gewinnen müssen, um Richtigschreibungen produzieren zu können. Die Qualität des Lernangebots wird nach Kriterien der Schriftsystematik und der Lernförderlichkeit erörtert (vgl. Corvacho del Toro, 2016b).

\section{Die Ebene der Lautstruktur}

\section{(1) Phonetik und Phonologie}

Alphabetische Schriftsysteme kodieren zunächst die Lautung (vgl. Thomé, 2000, 2019). Sie sind in der Regel phonembasiert, d.h. sie beziehen sich auf die Lautklassen einer spezifischen Sprache und bilden sie in der Reihenfolge des Vorkommens im Wort ab. Phoneme werden durch Grapheme verschriftlicht, die ihrerseits aus einem bis drei Buchstaben bestehen (vgl. Thomé, Siekmann et al., 2011). Welche 

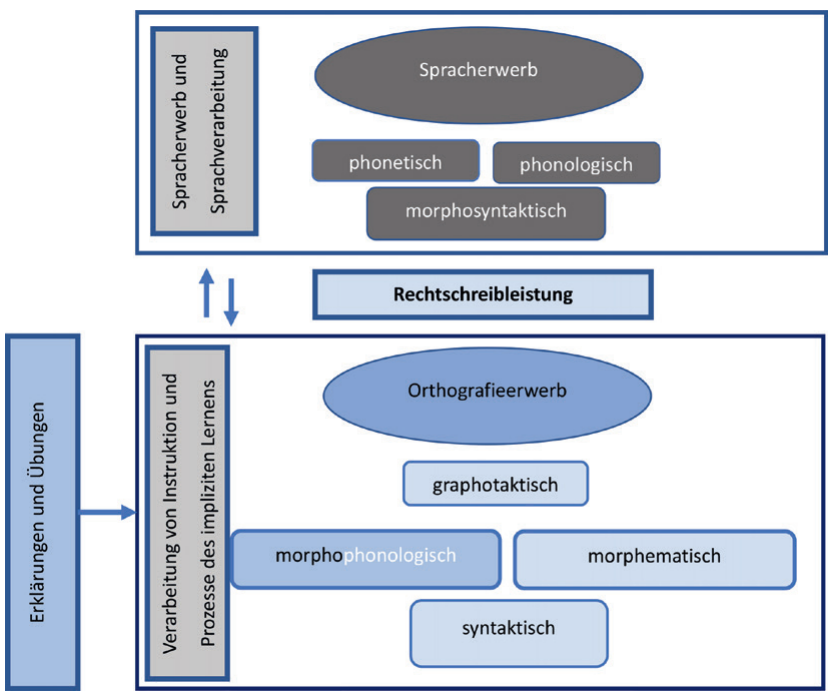

Abbildung 1. Linguistische und psycholinguistische Einflussfaktoren auf die Rechtschreibleistung. Abhängigkeit der Rechtschreibleistung von Spracherwerb und Sprachverarbeitung sowie von rechtschreibdidaktischer Instruktion und implizitem Lernen.

Sprachlaute als Lautklasse einer Sprache fungieren, wird durch Substitution ermittelt. Ergibt sich in einem Wortpaar durch Substitution eines Sprachlautes (bspw.: <packen> $<$ backen $>,<$ Rose $><$ Lose $>,<$ Wanne $><$ Wange $>$ ) eine neue Bedeutung, erweisen sich diese Sprachlaute als Phoneme (bspw.: /p/ und /b/, /r/ und /l/, /n/ und / $/$ /) der gegebenen Sprache. Beim Vokal Schwa verhält es sich so, dass es aufgrund von seiner eingeschränkten Stellung im Wort (nur in der unbetonten Silbe) und seiner eingeschränkten Funktion in der Fähigkeit zur Bedeutungsunterscheidung (meist in grammatischer Funktion bspw. <Stein>, <Steine> oder $<$ sein $>$, $<$ seine $>$ ) der Status als Phonem umstritten ist (vgl. Ternes, 2012). Wortpaare wie < braten $>$ und $<$ beraten $>$ erfüllen auch nicht die Kriterien phonologischer Minimalpaare: Die Anzahl der Phonem-Graphem-Korrespondenzen ist nicht identisch und im Wort $\{$ be $\}-\{$ raten\} liegt eine Morphemgrenze vor. In diesen Einschränkungen sind die linguistischen Wurzeln der Schwa-Tilgung zu sehen.

Die erste Herausforderung des Schriftspracherwerbs bzw. der Alphabetisierung besteht darin, aus dem Sprachfluss und den verschiedenen sprachsystematischen, sprachgemeinschaftlichen und individuellen Aussprachevarianten (Phone) die Lautklassen (Phoneme) der zu verschriftenden Sprache zu abstrahieren und mit einem entsprechenden Graphem wiederzugeben. Die Normierung der Aussprache und der Schrift als kulturelles Konstrukt und gesellschaftliche Übereinkunft (Konvention) erfordert das Erlernen einer neuen sog. Bildungssprache, welche die Standardlautung einschließt. Vorschulische Erfahrungen mitSchriftund Schriftmustern der Bildungssprache, die standardlautlich verfasst sind, spielen demnach eine bedeutende Rolle für den schulischen Schriftspracherwerb (vgl. Steinhoff, 2009).

\section{Meilenstein 1 - Abstraktion von Phonemen}

Auch wenn der erste Zugang zur Schrift die eigene Aussprache ist, so erweist sich die Aussprache bald als unzureichend, denn in der Schrift soll nicht die Aussprache, sondern eine konventionalisierte Abstraktion davon abgebildet werden. Diese erste Überlappung von Sprachebenen betrifft also die Bereiche der Phonetik (Aussprache) und Phonologie (systematische Laute einer Sprache) und bildet für die Schüler_innen den ersten großen Meilenstein (1) im Erwerb der Schriftsprache. Dieser Lernprozess wird durch die Schrift unterstützt. Die Schüler_innen lernen für jedes Phonem ein Graphem zu verschriften. Entsprechend der Systematik der deutschen Orthografie und unter Berücksichtigung lernförderlicher Kriterien sollte es das korrespondierende Basisgraphem sein, also das Schriftzeichen, das am häufigsten für das Phonem geschrieben wird (vgl. Thomé, Siekmann et al., 2011; Treiman \& Kessler, 2006; Corvacho del Toro 2016b). Diese Lautierkompetenz, d.h. das Erkennen und Identifizieren von isolierten Phonem-Graphem-Korrespondenzen, reicht aber nicht aus, um alphabetisch vollständige Wortschreibungen zu meistern (vgl. Corvacho del Toro \& Hoffmann-Erz, 2014, 2015).

\section{Meilenstein 2 - Unterschiede zwischen Standardlautung und Schrift}

Zur vollständigen Wiedergabe von Wörtern (alphabetisches Schreiben) müssen die Schüler_innen über die Abstraktion der Lautklassen hinaus systematische Unterschiede zwischen der Standardaussprache und der schriftlichen Wiedergabe von Wörtern berücksichtigen. Diese Hürde stellt die zweite Überlappung von Sprachebenen (Phonologie - Morphologie) dar, denn die Wiedergabe des Basisgraphems ist nun von der Stellung der Phonem-GraphemKorrespondenz im Wort abhängig (siehe Tab. 1).

Der erste zentrale Unterschied betrifft die Wiedergabe des Vokals Schwa (bspw. <Hose>, <selten>), der in der Aussprache oft getilgt wird. Schwa /ə/ ist der meistgebrauchte Vokal, denn er kommt in vielen Wortendungen vor. Er wird stets unbetont ausgesprochen und ist im Deutschen aufgrund seiner eingeschränkten Stellung im Wort nur bedingt bedeutungsunterscheidend. Die Tatsache, dass Unterrichtsmaterialien in der Regel das Schwa nicht explizit behandeln, verstärkt wahrscheinlich die Tilgung des Schwa in den Schülerschreibungen.

Der zweite systematische Unterschied zwischen der Standardaussprache und der schriftlichen Wiedergabe von Phonemen betrifft die vokalisierte Aussprache des Phonems $/ \mathrm{r} /$. Das Phonem $/ \mathrm{r} /$ hat eine konsonantische (bspw.: $<$ Raupe $>$ ) und eine vokalisierte Aussprache (bspw. $<$ Tür $>$ ). 
Tabelle 1. Meilensteine des Orthografieerwerbs

\begin{tabular}{|c|c|c|}
\hline $\begin{array}{l}\text { Linguistische } \\
\text { Disziplin }\end{array}$ & Meilensteine des Orthografieerwerbs & $\begin{array}{l}\text { Sprach-/ } \\
\text { Schriftebene }\end{array}$ \\
\hline \multirow[t]{5}{*}{ Syntax } & $\begin{array}{l}\text { Meilenstein } 11 \\
\text { Satzzeichen je nach Phrasen-/Satzstruktur (Interpunktion: Punkt, Komma, u.a.) }\end{array}$ & Textstruktur \\
\hline & $\begin{array}{l}\text { Meilenstein } 10 \\
\text { Wortgrenze je nach Phrasen-/Satzstruktur (Zusammen-/Getrenntschreibung von Nomen, } \\
\text { Verben, Präpositionen) }\end{array}$ & Satzstruktur \\
\hline & $\begin{array}{l}\text { Meilenstein } 9 \\
\text { Wortform je nach Satzstruktur (Konjunktionen mit Orthographemen) }\end{array}$ & \\
\hline & Meilenstein 8 & \\
\hline & Großschreibung je nach Phrasen-/Satzstruktur bestimmen (Nominalphrase) & \\
\hline Morphosyntax & $\begin{array}{l}\text { Meilenstein } 7 \\
\text { Wortform in Abhängigkeit von Rektion und Kongruenz bestimmen (grammatische Endungen) }\end{array}$ & \\
\hline \multirow[t]{2}{*}{ Morphologie } & $\begin{array}{l}\text { Meilenstein } 6 \\
\text { Phonem-(Ortho)graphem-Korrespondenz je nach Wortaufbau und/oder Wortbaustein bestimmen } \\
\text { (Zerlegen in Morpheme) }\end{array}$ & Wortstruktur \\
\hline & $\begin{array}{l}\text { Meilenstein } 5 \\
\text { Phonem-Orthographem-Korrespondenz je nach stammbildendem Morphem bestimmen } \\
\text { (Morphemkonstanz im weiteren Sinne) }\end{array}$ & \\
\hline $\begin{array}{l}\text { Morpho- } \\
\text { phonologie }\end{array}$ & $\begin{array}{l}\text { Meilenstein } 4 \\
\text { Phonem-Orthographem-Korrespondenz je nach Wortstruktur und Aussprache des betonten } \\
\text { Vokals bestimmen (Konsonantenverdopplung) }\end{array}$ & \\
\hline \multirow[t]{2}{*}{ Phonologie } & $\begin{array}{l}\text { Meilenstein } 3 \\
\text { Phonem-Orthographem-Korrespondenz je nach Abfolge von Phonemen und Stellung im Wort } \\
\text { bestimmen (eingeschränkte Graphemabfolgen) }\end{array}$ & \\
\hline & $\begin{array}{l}\text { Meilenstein } 2 \\
\text { Phonem-Basisgraphem-Korrespondenz in Abhängigkeit von der Stellung des Phonems im Wort } \\
\text { bestimmen (Schwa, r-Vokalisierung, Schwa }+/ r / \text { Abfolge) }\end{array}$ & Lautstruktur \\
\hline Phonetik & $\begin{array}{l}\text { Meilenstein } 1 \\
\text { Abstraktion einer Lautklasse (Phonem) aus der eigenen Aussprache (Phone), um es mit seinem } \\
\text { Basisgraphem abzubilden }\end{array}$ & \\
\hline
\end{tabular}

Anmerkung: Entsprechend den fachdidaktischen Kompetenzmodellen stehen die höheren Kompetenzstufen in der Tabelle oben.

Die jeweilige Aussprachevariante ist von der Stellung in der Silbe abhängig (Ternes, 2012). Silbenauslautend wird $/ \mathrm{r}$ / nicht konsonantisch, sondern wie ein Vokal [e] ausgesprochen. Bei Wörtern wie $<$ Tür $>$ ist das $<r>$ über $<$ Türe $>$ ableitbar, bei Wörtern wie $<$ Wurm $>$ müssen phonologische Übungen zur Unterscheidung der Aussprache mit und ohne $[\mathrm{e}]$ herangezogen werden, bei Wörtern wie <zart> entspricht die Wiedergabe des /r/ einer Merkleistung, denn nach /a/ ist die Vokalisierung [e] nicht wahrnehmbar. $\mathrm{Zu}$ diesem Bereich gehört auch die regelhafte Wiedergabe der Abfolge <er>, welche auch [e] ausgesprochen wird, in Wörtern wie $<$ Kinder, Bilder $>$. Meistern die Schüler_innen die Wiedergabe des Schwa, der Abfolge Schwa plus /r/-Phonem und des vokalisierten /r/Phonems, kann von einer vollentfalteten alphabetischen Schreibfähigkeit ausgegangen werden. Alle nach der Standardlautung im Wort vorkommenden Phoneme werden mit einem Basisgraphem abgebildet (vgl. Corvacho del Toro \& Hoffmann-Erz, 2014, 2015).

\section{Die Ebene der Wortstruktur}

Meilenstein 3 - Eingeschränkte Graphemabfolgen

Bei der dritten Überlappung im Modell lernen die Schüler_innen die ersten Orthographeme $(<\mathrm{s}>$ für $/ \mathrm{J} /$ und $<\mathrm{n}>$ für $/ \mathrm{y} /$ ) kennen. Diese Phonem-Graphem-Korrespondenzen sind in ihrem Vorkommen stellungsbedingt und umgebungsabhängig. Die Graphemabfolgen $<$ sp $>$, $<$ st $>$, $<$ qu $>$ kommen nur morpheminitial vor (in Wörtern wie $<$ Spiel $>$, $<$ Stein $>$, < Quark $>$ ). Die Graphemabfolge $<$ nk $>$ kommt nur silbenauslautend (in Wörtern wie <Anker $>$, <Dank $>$ ). Meistern die Schüler_innen diese Schreibungen kann von ersten orthografischen Einsichten, die sich von einer Wie- 
dergabe von Phonemen ausschließlich mit Basisgraphemen entfernt, ausgegangen werden. Sie identifizieren das Phonem, das Basisgraphem und untersuchen die Wortstruktur, um das Orthographem auszuwählen.

\section{(2) Morphophonologie}

Meilenstein 4 - Konsonantenverdopplung

Die Markierung der Vokalkürze durch die Verdopplung des Folgekonsonanten stellt die vierte Überlappung dar. Zur korrekten Markierung der Vokalkürze durch Konsonantenverdopplung (bspw.: <Wolle>) muss neben dem Phonem $/ \mathrm{o} /$ ) die Wortstruktur des stammbildenden Morphems berücksichtigt werden (vgl. Eisenberg \& Fuhrhop, 2007). Wörter wie <Wolke $>$ erfordern keine Konsonantenverdopplung, trotz des kurzen Vokals. Bei der Wortstruktur mit Konsonantenverdopplung $<$ Hölle $>$ führt die Leseprobe zum Ergebnis. Wird das Wort ohne Konsonantenverdopplung $\left({ }^{*}<\right.$ Höle $>$ ) geschrieben, ist die Leseart mit lang ausgesprochenem Vokal vorgeschrieben und das Wort in seiner Lautung verfälscht. Das Wort ${ }^{*}<$ Wole $>$ entspräche wortstrukturell Wörtern wie $<$ Hose, Lupe, Schal, Löwe $>$ und wäre in seiner Lautung verfälscht (vgl. $<$ Wolle $>,<$ Schall $>$ ). Es ist also die schriftliche Wortstruktur, die die Wortlautung hervorbringt und die Notwendigkeit der Markierung anzeigt. Der Kontrast zwischen stammbildenden Morphemen mit und ohne Konsonantenverdopplung wird zur Einsicht in die Markierung der Vokalkürze empfohlen (vgl. Corvacho del Toro, 2016c, 2019). Der wortstrukturelle Ansatz setzt auf die Ableitung über die stammbildenden Morpheme. Wörter wie <rufst> oder $<$ trennbar $>$, die durch Komposition oder Derivation gebildet werden, erlauben keinen direkten Vergleich mit den zentralen vier Mustern der Markierung der Vokalquantität (unmarkiert: <Wolke>, markiert: <Himmel > sowie unmarkiert: $<$ Hose /Liebe $>$, markiert: $<$ bohren $>$ ). Erst die stammbildenden Morpheme $<$ rufen $>$ und $<$ trennen $>$ geben Klarheit.

Wörter mit den Graphemen <sch, ch, ng, pf, chs> (bspw.: $<$ Flasche, Buch, Schlange, Napf, Lachs $>$ ) können bei dieser Betrachtung vernachlässigt werden, denn Grapheme, die aus mehreren Buchstaben bestehen, werden nie verdoppelt. Rechtschreibfehler aufgrund von verdoppelten Graphemen für <sch, ch, ng, pf, chs> sind in ausgewerteten Fehlerkorpora bislang nicht vorgekommen. Die Frage nach der Vokalkürze oder -länge bei Wörtern mit <sch, ch, ng, pf, chs > erübrigt sich, zumindest im Hinblick auf die Rechtschreibung.

Schüler_innen, die den Unterschied zwischen den langen und kurzen Vokalen nicht wahrnehmen können, lernen die Markierungen der Vokalkürze als feststehendes Morphem (bspw.: \{Blatt, still, müss-en\} usw.) kennen. Am besten in Analogiebildung zu anderen Wörtern mit der gleichen Wortstruktur. Dieser fachdidaktische Ansatz wäre ein rein morphologischer, der fehlende phonologische Unterscheidung kompensiert.

\section{(3) Morphologie}

\section{Meilenstein 5 - Morphemkonstanz im weiteren Sinne}

Die Morphemkonstanz in der deutschen Orthografie berührt viele orthografische Phänomene (vgl. Corvacho del Toro, 2017; Nerius 2007, Elsen, 2014). Bewusst sind den meisten Lehrkräften und Schüler_innen vor allem die konsonantischen und vokalischen Ableitungen, die die Morphemkonstanz bedingt: Die Orthographeme $<b, d, g>$ am Silbenende in Wörtern wie $<$ Raub, Hund, Berg $>$ sowie die Orthographeme <ä, äu $>$ bei Wörtern, die sich aus einer Grundform mit $<$ a, au $>$ ableiten $(<$ Äpfel, Mäuse $>$ ). Die Morphemkonstanz berührt aber auch die Markierung der Vokallänge mit <ah, uh, eh, üh> u.a. und der Vokalkürze mit $<\mathrm{pp}, \mathrm{ck}$, tt, mm, tz> u.a., indem sie diese Markierung auf abgeleitete Wörter überträgt. Bleibt die Vokalquantität (kurzer / langer Vokal) in den abgeleiteten Wörtern unverändert, wird die Vokalkürze- oder Längenmarkierung in den abgeleiteten Wörtern übernommen. Die Morphemkonstanz führt somit neben ihrer wichtigen Funktion der Leseerleichterung zur großen Rechtschreibsicherheit (vgl. Galuschka et al., 2020; Görgen et al., 2021; Volkmer et al., 2019; Kirby et al., 2012). Die Ausnahmen sind wenige und können als Merkschreibungen behandelt werden (bspw.: $<$ kommen $>$ aber $<$ kam $>$ ). Einige eignen sich, innerhalb eines Musters (bspw.: <schließen, schloss, geschlossen>, $<$ schießen, schoss, geschossen>) erlernt zu werden.

Während die Markierung der Vokalkürze innerhalb des stammbildenden Morphems regelhaft konstruierbar und durch die Leseprobe $\left({ }^{*}<\right.$ Wole $>$ oder $<$ Wolle $\left.>\right)$ überprüfbar ist (vgl. Thelen, 2002), stellt für die Markierung eines Langvokals die Vokallänge zwar eine Bedingung, aber keine Regel für das Vorkommen des $h$ in Graphemen wie $<$ ah, uh, öh $>$ u.a. oder die Vokal-Doppelschreibung $(<\mathrm{aa}\rangle,\langle 00\rangle$, $<$ ee>) dar (vgl. Eisenberg \& Fuhrhop, 2007; Corvacho del Toro, 2016c, 2019). Daher führt die Befolgung der Regel „Nach Langvokal schreibt man h“ o.̈̈. zu vielen Rechtschreibfehlern. Systematischer ist es, sich die stammbildenden Morpheme mit h-Markierung \{fahr, zahl, wohn\} und Doppelvokal \{Saal, Moor, See\} zu merken und dann abgeleitete Wörter mit diesen Morphemen zu erkennen und entsprechend der Grundform zu schreiben (bspw.: <fahren, Fahrer, Fahrerlaubnis, Gefahr> u.a.) (vgl. Corvacho del Toro, 2019). Diese Aufgabe ist zu bewältigen, denn die stammbildenden Morpheme mit $h$ und Doppelvokal sind in der Anzahl gering. Andererseits sind sie durch die Morphemkonstanz sehr ergiebig (vgl. Augst, 1998; Elsen, 2014).

Grundsätzlich wird empfohlen, zuerst das Schreiben von stammbildenden Morphemen und anschließend das Schreiben von abgeleiteten Formen (bspw.: <Himmel> dann $<$ himmelblau, himmlisch, anhimmeln $>$ ) einzuführen. Die- 
ses Vorgehen fördert die Entwicklung morphematischer Bewusstheit und ist aufgrund des Aufbaus der deutschen Orthografie zielführend in der Rechtschreibdidaktik. Die Kenntnis der vier verschiedenen Wortstrukturen der stammbildenden Morpheme (unmarkiert: <Wolke>, markiert: <Himmel> sowie unmarkiert: <Hose/Liebe>, markiert: $<$ bohren>) ist grundlegend (Corvacho del Toro, 2019).

\section{Meilenstein 6 - Wortaufbau durchschauen}

An dieser Stelle kommt die sechste Überlappung im orthografischen System hinzu: Eine Phonem-Graphem-Korrespondenz wird je nach Wortaufbau wiedergegeben. Bei zusammengesetzten Wörtern ist die Morphemgrenze entscheidend: $<$ Hand $>$ und $<$ Tuch $>$; $<$ Handtuch $>$. Bei diesem und ähnlichen Beispielen führt das Wissen um den Wortaufbau zur Richtigschreibung. Bei Nicht-Berücksichtigung des Wortaufbaus sind Schreibungen wie ${ }^{*}<$ Hantuch $>$, * $<$ entäuscht $>$ erwartungsgemäß. In der Diagnose und Förderung sind diese Fehler strikt von Rechtschreibfehlern der echten Phonemauslassung wie ${ }^{*}<$ schechter $>$ für $<$ schlechter $>$ ) oder Phonemverwechslung wie ${ }^{*}<$ Bost $>$ für $<$ Post $>$ zu unterscheiden (Corvacho del Toro, 2016b), denn an der Morphemgrenze sind nacheinander vorkommende Plosive (/p, t, k/) in natürlicher Aussprache im Gegensatz bspw. zu Lateralen <Ziellinie> nicht wahrnehmbar. Die Fähigkeit, Wörter in ihre Morpheme zu zerlegen, ist ebenso für das Erkennen und das richtige Schreiben von Affixen, d.h. Wortbausteine wie \{ver, ab, ig\} in Wörtern wie $<$ Lehrveranstaltung, abfahren, Müdigkeit>, erforderlich. Im Fokus der Instruktion stehen der Wortaufbau und die Schreibung von Wortbausteinen, vor allem von denen, die ein Orthographem enthalten.

\section{Die Ebene der Phrasen-/Satzstruktur}

\section{(4) Morphosyntax}

Meilenstein 7 - grammatische Endungen

und Übereinstimmung

Morphosyntaktische Fähigkeiten betreffen die Übereinstimmung (Kongruenz) zwischen Subjekten und Prädikaten im Satz (bspw.: <ich esse, du singst $>$ ) sowie zwischen Prädikaten (bspw.: <Ich esse nur einen Salat>) und / oder Präpositionen und den sog. Falldeklinationen (Rektion) (bspw.: $<$ mit einem schönen Lächeln > oder < für gute Zwecke $>$ ). Es geht also um die Wortform in Abhängigkeit von Rektion und Kongruenz. Diese kann nur unter Berücksichtigung ei-

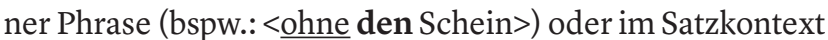
erfolgen. Der Erwerb des komplexen Konjugations- und Deklinationssystems des Deutschen ist Bestandteil des Spracherwerbsprozesses (Ulrich, 2018; Fischer \& Mollica, 2012). Diskrepanzen zwischen der bildungssprachlich normierten grammatischen Korrektheit und/oder Angemes- senheit und der konkreten Anwendung von Sprache im Alltag stellt auch für Schüler_innen mit Erstsprache Deutsch eine Herausforderung dar. Es sind aber die Schüler_innen mit einer grammatischen Störung oder Deutsch als Zweitoder Fremdsprache, die persistierende Schwierigkeiten vor allem beim Deklinationssystem zeigen (vgl. Berg, 2005; Aguado, 2012). In diesem Sinne nimmt dieser Meilenstein eine besondere Stellung ein. Grammatische Fehler werden in der Schule gesondert betrachtet und behandelt, in der Regel gehören sie nicht zum Kompetenzbereich Texte schreiben. Das Schreiben von Texten stellt aber das Ziel des Rechtschreibunterrichts oder einer Rechtschreibförderung dar, so gehören die Überprüfung der grammatischen Korrektheit sowie grammatische Instruktion zum Lernangebot. Ohnehin sind Rechtschreibung und Grammatik eng miteinander verzahnt. Die Förderung grammatischer Fähigkeiten erfordert ein ähnliches sprachanalytisches und strukturelles Vorgehen wie die Rechtschreibförderung, bei der Kriterien der Regelhaftigkeit, Ableitbarkeit, statistische Vorkommenshäufigkeiten und Merkleistungen eine tragende Rolle spielen.

Meilenstein 8 - Großschreibung je nach Phrase oder Satz

Die satzinterne Großschreibung, d.h. die Großschreibung von Nomen bzw. von Kernen von Nominalphrasen $(<$ die gute Luft $>$, <verpasste Gelegenheiten>), ist eine Eigenheit des Deutschen. Sie kann phrasenstrukturell beschrieben und gelernt werden. Großgeschrieben wird der erste Buchstabe des Wortes bzw. des Graphems (<im Eifer >, <beim Niesen $>$ ). Das Erlernen der Großschreibung beginnt in der Grundschule, dabei hat sich die Analyse der Nominalphrase als effektiver didaktischer Ansatz erwiesen (Brucher, Ugen \& Weth, 2020; Röber-Siekmeyer, 1999). Zusätzlich zu der Erweiterungsprobe (d.h. ob das Nomen durch Linksattribute beschrieben werden kann: <das vereinbarte Treffen $>$, <gute Gründe $>$ ) soll die Aufmerksamkeit auf die Deklinationen gelenkt werden (vgl. Funke, 2017). Die strukturelle Durchdringung der Nominalphrase mit den Deklinationen bietet eine effektive Orientierung zur Bestimmung der Zusammenschreibung von Adjektiven $\left({ }^{*}<\right.$ der nass kalte Winter $>$ statt $<$ der nasskalte Winter $\left.>\right)$ und von Nomen $\left({ }^{*}<\right.$ keine andere Auto Werkstatt $>$, $<$ keine andere Autowerkstatt $>$ ). Die Überprüfung der Bezüge zwischen Nomen und Adjektiven führt zur richtigen $\mathrm{Zu}$ sammenschreibung (vgl. Fuhrhop, 2010). Bereiche der Großschreibung wie die Großschreibung von Adjektiven (bspw. <etwas Kleines> oder < für Jung und Alt>) können aus statistischer und schriftsystematischer Perspektive als Besonderheit betrachtet werden. Sie sollten getrennt vom regelhaften Bereich der satzinitialen und satzinternen Großschreibung behandelt und ebenfalls im Phrasen-/ Satzzusammenhang überprüft werden. 
Meilenstein 9 - Wortform je nach Satzstruktur

Die Schreibung der Konjunktion $<$ dass $>$ mit dem Orthographem <ss> hängt von der Satzstruktur ab (vgl. Pittner \& Bermann, 2015; Granzow-Emden, 2013). An dieser Schreibung wird die Zugehörigkeit zu einem orthografischen Phänomen, nämlich der Konsonantenverdopplung, aufgrund seiner grafischen Form (das Graphem $<$ ss $>$ ) getroffen. Auch wenn auf graphematischer Ebene die Konsonantenverdopplung das Merkmal ist, gehört die Richtigschreibung zu einem anderen Lernbereich. Erst die Analyse des Satzes und die Einsicht, dass es sich um eine Konjunktion handelt, führt zur Richtigschreibung. Der Lernweg kann aber implizit unterstützt werden, indem die Schüler_innen musterhafte Sätze mit <dass> produzieren und sich auch über weitere Merkmale dieser Konstruktion, wie die Verben, die sie anleiten, bewusst werden.

Meilenstein 10 - Wortgrenze je nach Phrase oder Satz Der zehnte Meilenstein betrifft die Kompetenzen im Bereich des Wortkonzepts. Ein ausgebildetes Wortkonzept äußert sich darin, dass die Wortgrenzen stets eingehalten werden. Was bereits zu Anfang des Schriftspracherwerbs eher implizit gelernt wird, bleibt bis ins Erwachsenenalter ein fehlerträchtiger Bereich der deutschen Orthografie. Nach der Einhaltung von Spatien zwischen Wörtern folgt die Zusammenschreibung von Komposita (<Haustür>, $<$ nasskalt>, <himmelblau >), dann die Zusammenschreibung von trennbaren Verben in den Infinitiven und Partizipien (<ausleihen>, <abzugeben>, <abgefahren $>$ ). Diese Zusammenschreibungen sind charakteristisch für das deutsche Schriftsystem und verlangen satzanalytische Fähigkeiten (vgl. Fuhrhop, 2010). Kommen die Bestandteile der Zusammensetzungen frei vor, ist die Entscheidung über die verbindliche Zusammenschreibung zu begründen (bspw. $<$ mithilfe $>$, <indem>).

\section{Die Ebene der Textstruktur}

\section{(5) Syntax}

Meilenstein 11 - Satzzeichen je nach Phrase oder Satz

Die Satzzeichen, Punkt, Komma, Doppelpunkt, Anführungszeichen u.a., gehören in der Schule zu einer eigenen Fehlerkategorie, den Zeichensetzungsfehlern. In einer umfassenden theoretischen und fachdidaktischen Konzeption der deutschen Rechtschreibung und des Recht- / und Textschreibens müssen sie berücksichtigt werden (vgl. Nerius, 2007). Strukturell betrachtet besteht die einfachste Vorgabe darin, vollständige Sätze (d.h. sie beinhalten ein Subjekt und ein Prädikat) durch Satzzeichen getrennt voneinander zu schreiben. So müssen Schüler_innen zu einem strukturell überprüfbaren Konzept vom vollständigen Satz gelangen, bevor sie in der Lage sind, vollständige Sätze durch Satzzeichen
(Komma, Punkt u.a.) zu kennzeichnen. Hierfür müssen die Satz- sowie die Klammerstruktur des deutschen Satzes explizit gelernt, erkannt und in Texten produziert werden.

\section{Ebenenübergreifend}

\section{(6) Lexik}

Merkleistungen

Grundsätzlich wird bei dieser Modellierung zwischen dem Kernbereich und der Peripherie des Wortschatzes unterschieden. Es wird empfohlen, auch den Schüler_innen diese Unterscheidung nahezubringen. Erfahrungen in verschiedenen Schulklassen, auch der Grundschule, zeigen, dass dies möglich und effektiv ist. Ohne etymologische Kenntnisse können Schüler_innen anhand der Struktur von Wörtern diese als nativ oder fremder Herkunft kategorisieren: Betonungsmuster, Silbenanzahl, Vorkommen vom Schwa-Vokal (vgl. Nübling, 2008). Durchgehend wird beim vorgestellten rechtschreibdidaktischen Ansatz zwischen regelhaften Bereichen, die anhand von erkennbaren und sich wiederholenden Mustern gelernt werden können, und nicht regelhaften Schreibungen, die als Merkleistungen mit Hilfe von memorierenden Techniken gelernt werden müssen, unterschieden. Merkleistungen betreffen alle Stufen des Schriftsprach- und Orthografieerwerbs. Es beginnt mit dem Schreiben von Funktionswörtern wie $<$ und $>$, dem vokalisiertem $/ \mathrm{r} /$ nach $<\mathrm{a}>(<$ Bart $>)$, nicht ableitbaren Wörtern mit Orthographemen ( $<$ Säule $>$ oder $<$ Stadt $>$ ) und reicht bis in die höheren Kompetenzstufen des Orthografieerwerbs bei Schreibungen aus dem Bereich der Zusammen- und Getrenntschreibung <das Gewusst-wie>) (vgl. Thomé \& Eichler, 2008; Naumann, 2006).

\section{Zusammenfassung der Ergebnisse}

Die Beschreibung der Meilensteine zeigt die Überlappungen zwischen den linguistischen Bereichen (Phonetik und Phonologie, Phonologie und Morphologie, Morphologie und Syntax sowie Syntax und Textualität). Darüber hinaus wird deutlich, dass die Wortstruktur bereits nach dem ersten Meilenstein im Zentrum der Analyse steht, und es bis Meilenstein 10 bleibt (siehe Tab. 1).

Aus der erarbeiteten Systematik heraus wird zum Erreichen einer großen Rechtschreibsicherheit empfohlen, zunächst das Erlernen der Phonem-Basisgraphem-Korrespondenzen einzuführen $(90 \%$ der Grapheme eines Textes). Da das Vorkommen der Orthographeme (insgesamt $10 \%$ der Grapheme eines Textes) sich erst über die Analyse der Wortstruktur und des Wortaufbaus bestimmen lässt, sollen diese eine zentrale Stellung in der Rechtschreibdidaktik annehmen. Bereits ab dem Meilenstein 2 
(im Bereich der Lautstruktur) spielt die Wortstruktur eine gewichtige Rolle, denn sie muss berücksichtigt werden, um zur Richtigschreibung von Basisgraphemen (das Schwa, das vokalisierte R und die Abfolge Schwa + R) zu gelangen. Diese zentrale Stellung des Wortes (Wortstruktur/Wortaufbau) durchzieht sich bis Meilenstein 10 innerhalb von Phänomenen der Laut-, Wort- und der Satzebene. Bei den Phänomenen der Satzstruktur verhält es sich so, dass zwar die Phrase oder der ganze Satz Gegenstand der Analyse ist, diese Analyse findet aber statt, um die Wortform bestimmen zu können (satzinterne Großschreibung, Wortgrenzen, Wortendungen). Erst innerhalb der Textebene büßt das Wort etwas von seiner zentralen Stellung ein, denn hier wird die Aufmerksamkeit auf die Analyse des Satzes gerichtet und ihr Resultat beeinflusst nur bedingt die Wortform (satzinitiale Großschreibung).

Als Produkt psycholinguistischer Spracherwerbs- und Sprachverarbeitungsprozesse und als zentraler Befund bei der Feststellung einer Rechtschreibstörung stehen Rechtschreibfehler im Fokus psycholinguistischer und rechtschreibdidaktischer Untersuchungen. Auch bei diesem Beitrag gilt es zu betonen, dass die vorgestellten Meilensteine keine Linearität darstellen, da sie auf Fehlerverteilungen basieren, die stets hierarchisch parallel in Beziehung zueinander stehen (Eichler, 1986), d.h. erst die prozentualen Verteilungen verschiedener Fehlertypen erlauben eine zuverlässige Aussage über den Lernstand. Gerade zu Anfang einer Förderung und bei einer hohen Anzahl von Rechtschreibfehlern sind Fehler aller Art in einer Schreibprobe zu finden. Erst nach einer systematischen Förderung können gesicherte Hinweise über persistierende Fehlertypen getroffen werden. Während sich bestimmte Fehlerarten stark reduzieren und überwunden werden, persistieren andere und müssen kontinuierlich Bestandteil des Lernangebots sein. Ausgehend von dieser theoretischen Modellierung wird angenommen, dass Rechtschreibfehler hauptsächlich phonologisch, morphosyntaktisch (grammatisch) oder morphologisch begründet sind. Die Identifizierung dieser Bereiche als ursächlich für (Rechtschreib)fehler soll weitere Klarheit für die Diagnose und Förderung der Rechtschreibstörung bringen.

\section{Diskussion}

\section{Diskussion der Ergebnisse}

Der vorliegende Beitrag hatte zum Ziel eine linguistisch fundierte und fachdidaktisch orientierte Darstellung des Erwerbs der deutschen Orthografie aufzuzeichnen. Dieser verdeutlicht und zeigt in schematischer Weise die Meilensteine des Orthografieerwerbs aus linguistischer und psycholinguistischer Perspektive. Die Arbeit plädiert für die
Fokussierung auf Wortstrukturen und Wortaufbau in der Rechtschreibdidaktik und Rechtschreibförderung. Die zentrale Stellung des Wortes als Analyseeinheit bei der Entscheidung über Phonem-Graphem-Korrespondenzen begründet diese Position. Die für die Lehrerfortbildungsund Interventionsstudie entwickelten Fortbildungs- und Unterrichtsmaterialien (siehe Forschungsrahmen) tragen dieser Systematik Rechnung.

Des Weiteren bietet dieses theoretische Modell eine linguistische Grundlage für eine differenzierte Betrachtung der Symptomatik der Rechtschreibstörung und zur Annahme verschiedener Subtypen von Rechtschreibstörungen: 1) phonologisch 2) morphosyntaktisch (grammatisch) und 3) morphologisch. Rechtschreibfehler, die persistierend phonologische und morphosyntaktische Schwierigkeiten widerspiegeln, könnten ihre Ursache im Spracherwerbs- und Sprachverarbeitungsprozess haben, während Fehler, die auf morphologische und syntaktische Fähigkeiten verweisen, Fragen über Beeinträchtigungen des impliziten Lernens (Ise \& Schulte-Körne, 2012) und / oder die Qualität und Intensität von Instruktion und Übungsangeboten aufwerfen (siehe Abb. 1).

Entsprechend wären die Subtypen der Rechtschreibstörung ein phonologischer und/oder morphosyntaktischer Subtyp, bei dem Spracherwerbs- und Sprachverarbeitungsprozesse im Vordergrund stehen, und ein morphologischer, syntaktischer Subtyp, bei dem das implizite Lernen bzw. die Beeinträchtigung des impliziten Lernens und/oder die Qualität der Instruktion ursächlich für die Rechtschreibschwierigkeiten sind.

\section{Relevanz für die Praxis}

Die Effektivität eines Lernangebots oder einer Rechtschreibförderung hängt maßgeblich von der fachdidaktischen Qualität und der Passung zum Lernstand des Schülers oder der Schülerin ab. Dafür ist es notwendig, dass Lehrkräfte und Lerntherapeut_innen über theoretische Klarheit im Hinblick auf Gestalt und Systematik der deutschen Orthografie sowie dem Erlernen der Rechtschreibung verfügen. Dieser Beitrag möchte mit einer theoretischen Modellierung zu einem tieferen Verständnis der deutschen Orthografie und zu einer verbesserten sprachdidaktischen Qualität von Unterstützungsangeboten beitragen. Erste Unterrichtsmaterialien, die auf dieser Konzeption basieren, werden in Projektschulen eingesetzt.

\section{Limitationen}

Die vorgestellte theoretische Arbeit entstand im Rahmen einer Lehrerfortbildungs- und Interventionsstudie. Eine 
statistisch gesicherte Wirksamkeit der vorgestellten Lernprogression und der empfohlenen Instruktion (Basisgrapheme, Wortstrukturen, Wortaufbau, Nominalphrase u.a.) durch ein Experimental-/Kontrollgruppendesign wurde im Schuljahr 2019/20 gestartet. Die Studie kam durch die Corona-Pandemie im März 2020 zum Abbruch, sodass der Beitrag sich auf die theoretischen Grundlagen beschränken muss. Eine empirische Überprüfung der Wirksamkeit einer Intervention auf Grundlage dieser theoretischen Modellierung steht aus.

\section{Literatur}

Aguado, K. (2012). Progression, Erwerbssequenzen und Chunks. Zur Lehr- und Lernbarkeit von Grammatik im Fremdsprachenunterricht. AkDaF Rundbrief, 64, 7-22.

Augst, G. (1974). Rechtschreibung mangelhaft? Heidelberg: Quelle \& Meyer.

Augst, G. (1998). Wortfamilienwörterbuch der deutschen Gegenwartssprache. Tübingen: Niemeyer.

Berg, M. (2005). Förderung komplexer syntaktischer Fähigkeiten spracherwerbsgestörter Kinder. Dissertation. Pädagogische Hochschule Heidelberg. http://www.ub.uni-heidelberg/archiv/6209

Bredel, U. (2006). Die Herausbildung des syntaktischen Prinzips in der Historiogenese und in der Ontogenese der Schrift. In U. Bredel \& H. Günther (Hrsg.), Orthographietheorie und Rechtschreibunterricht (S.139-164). Tübingen: Niemeyer.

Bredel, U. (2010). Die satzinterne Großschreibung - System und Erwerb. In U. Bredel, A. Müller \& G. Hinney (Hrsg.), Schriftsystem und Schrifterwerb (S.217 - 234). Berlin u.a.: De Gruyter.

Brucher, L., Ugen, S. \& Weth, C. (2020). The impact of syntactic and lexical trainings on capitalization of nouns in German in grade five. L1-Educational studies in Language and Literature, 20, $1-23$.

Clahsen, H. \& Fleischhauer, E. (2014). Morphological Priming in Child German. Journal of Child Language, 41, 1305-1333.

Corvacho del Toro, I. (2016a). Sprachliche Heterogenität und individuelle Lernunterstützung. Über die Relevanz des Fachwissens und des fachdidaktischen Wissens im mehrsprachigen Kontext. Leseräume, 3, 84-97.

Corvacho del Toro, I. (2016b). Zur qualitativen Rechtschreibfehleranalyse und einer schriftsystematischen lernförderlichen Behandlung der Rechtschreibstörung. Zeitschrift für Kinder- und Jugendpsychiatrie und Psychotherapie, 44, 397- 408.

Corvacho del Toro, I. (2016c). Zweisilber - Ja! Silbentrennung Nein! Zweisilber im alphabetischen Schreiben und in der Konsonantenverdopplung. Grundschulunterricht Deutsch, 3, $36-38$.

Corvacho del Toro, I. (2017). Orthographische Prinzipien aus graphematischer Sicht. Eine neue Betrachtung. In K. Siekmann, I. Corvacho del Toro \& R. Hoffmann-Erz (Hrsg.), Schriftsprachliche Kompetenzen in Theorie und Praxis (S. 61 - 70). Tübingen: Stauffenburg.

Corvacho del Toro, I. (2019). „Die Mittelstufe schreibt!“ Lehrmaterialien für den Rechtschreibunterricht der Sekundarstufe I. Verfügbar unter https://www.deutschland-schreibt.de/downloads/

Corvacho del Toro, I. \& Hoffmann-Erz, R. (2014). Was ist lautgetreu? Zur Notwendigkeit einer begrifflichen Differenzierung. In K. Siekmann (Hrsg.), Theorie, Empirie und Praxis effektiver Rechtschreibdiagnostik (S. 29 - 40). Tübingen: Stauffenburg.
Corvacho del Toro, I. \& Hoffmann-Erz, R. (2015). Liegt die „Rechtschreipkaterstrofe" an der Lautorientierung? Grundschulzeitschrift, 290, $36-39$.

Dahmen, S. (2012). Orthographiefehler bei DaZ-Lernern - Ursachen, Diagnostik und Training. In M. Michalak \& M. Kuchenreuther (Hrsg.), Grundlagen der Sprachdidaktik Deutsch als Zweitsprache (S.143-162). Baltmannsweiler: Schneider Hohengehren.

Eichler, W. (1986). Zu Uta Frith' Dreiphasenmodell des Lesen (und Schreiben) Lernens. Oder: Lassen sich verschiedene Modelle des Schriftspracherwerbs aufeinander beziehen und weiterentwickeln? In G. Augst (Hrsg.), New Trends in Graphemics and Orthography (S.234-237). Berlin u.a.: de Gruyter.

Eisenberg, P. (2017). Deutsche Orthografie. Regelwerk und Kommentar. Berlin: De Gruyter.

Eisenberg, P. \& Fuhrhop, N. (2007). Schulorthographie und Graphematik. Zeitschrift für Sprachwissenschaft, 26, 15-41.

Elsen, H. (2014). Grundzüge der Morphologie des Deustschen (2., aktualisierte Aufl.). Berlin, u.a.: De Gruyter.

Fischer, K. \& Mollica, F. (2012). Valenz, Konstruktion und Deutsch als Fremdsprache. Frankfurt am Main u. a.: Peter Lang.

Fuhrhop, N. (2010). Getrennt- und Zusammenschreibung: Kern und Peripherie. Rechtschreibdidaktische Konsequenzen aus dieser Unterscheidung. In U. Bredel, A. Müller \& G. Hinney (Hrsg.), Schriftsystem und Schrifterwerb (S.235-258). Berlin: De Gruyter.

Funke, R. (2017). Syntaxbasierte Vermittlung der satzinternen Großschreibung: Varianten eines Ansatzes. In I. Rautenberg \& S. Helms (Hrsg.), Der Erwerb schriftsprachlicher Kompetenzen (S.100 - 120). Baltmannsweiler: Schneider Verlag Hohengehren.

Galuschka, K., Görgen, R., Kalmar, J., Haberstroh, S., Schmalz, X. \& Schulte-Körne, G. (2020). Effectiveness of spelling interventions for learners with dyslexia: A meta-analysis and systematic review. Educational Psychologist, 55(1), 1 - 20. https://doi.org/10 .1080/00461520.2019.1659794.

Görgen, R., De Simone, E., Schulte-Körne, G. \& Moll, K. (2021). Predictors of reading and spelling skills in German: The role of morphological awareness. Journal of Research in Reading. https:// doi.org/10.1111/1467-9817.12343

Granzow-Emden, M. (2013). Deutsche Grammatik verstehen und unterrichten. Tübingen: Niemeyer.

Hoffmann-Erz, R. (2015). Lernprozesse im Orthographieerwerb. Eine empirische Studie zur Entwicklung der Generalisierungskompetenz. Berlin: wvb.

Ise, E., Schulte-Körne, G. (2012). Implizites Lernen und LRS. Lernen und Lernstörungen, 1,79-97.

Jakobson, R. (1969). Kindersprache, Aphasie und allgemeine Lautgesetze. Frankfurt am Main: Suhrkamp.

Kargl, R. \& Landerl, K. (2018). Beyond Phonology. The role of morphological and orthographic spellings in German. Topics in Language Disorders, 38 (4), 272 - 285. https://doi.org/10.1097/ TLD.0000000000000165

Kargl, R., Wendtner, A., Purgstaller, C. \& Fink, A. (2018). Der Einfluss der morphematischen Bewusstheit auf die Rechtschreibleistung. Lernen und Lernstörungen, 7, 45-54. https://doi.org/ 10.1024/2235-0977/a000202

Kirby, J. R., Deacon, S. H., Bowers, P. N., Izenberg, L., Wade-Woolley, L. \& Parrila, R. (2012). Children's morphological awareness and reading ability. Reading and Writing, 25, 389-410. https://doi. org/10.1007/s11145-010-9276-5

Munske, H. H. (2005). Lob der Rechtschreibung. Warum wir schreiben, wie wir schreiben. München: Beck.

Naumann, C. L. (2006). Rechtschreiberwerb. Die graphematischen Grundlagen und eine Modellierung bis zum Ende der Schulzeit. In S. Weinhold (Hrsg.), Schriftspracherwerb empirisch. Konzepte Diagnostik - Entwicklung (S.45-86). Baltmannsweiler: Schneider Verlag Hohengehren. 
Nerius, D. (Hrsg.) (2007). Deutsche Orthographie (4., neu bearb. Aufl.). Hildesheim: Olms.

Nübling, D. (2008). Historische Sprachwissenschaft des Deutschen. Eine Einführung in die Prinzipien des Sprachwandels (2., überarbeitete Aufl., mit A. Dammel, J. Duke, R. Szczepaniak). Tübingen: Günter Narr.

Pittner, K. \& Bermann, J. (2015). Deutsche Syntax. (6. Auflage). Tübingen: Narr.

Röber-Siekmeyer, C. (1999). Ein anderer Weg zur Groß- und Kleinschreibung. Leipzig: Klett Grundschulverlag.

Scheerer-Neumann, G. (2014). Punktgenau fördern! Warum? Ein historischer Rückblick und einige Daten. In G. Schulte-Körne \& G. Thomé (Hrsg.), LRS - Legasthenie: interdisziplinär (S.115-135). Oldenburg: isb.

Siekmann, K. \& Corvacho del Toro, I. (2018). Individuelle Förderung bei Deutsch als Zweitsprache. Grundschule, 6, 33-35.

Siekmann, K. \& Thomé, G. (2018). Der orthographische Fehler. Grundzüge der orthographischen Fehlerforschung und aktuelle Entwicklungen (2., aktualisierte Aufgabe). Oldenburg: isb Verlag.

Steinhoff, T. (2009). Wortschatz - eine Schaltstelle für den schulischen Spracherwerb? In H. Feilke, K.-P. Kappest \& C. Knoblauch (Hrsg.), Siegener Papiere zur Aneignung sprachlicher Strukturformen, 17, 1-63.

Ternes, E. (2012). Einführung in die Phonologie. (3., überarbeitete Auflage). Darmstadt:WBG.

Thelen, T. (2002). Wie passt das Wort BETTEN in das Haus? Grundlagen und Ergebnisse des Computerprogramms MoPs zur Vermittlung der Schärfungsschreibung. In D. Tophinke \& C. RöberSiekmeyer (Hrsg.), Schärfungsschreibung im Fokus. Zur schriftlichen Repräsentation sprachlicher Strukturen im Spannungsfeld von Sprachwissenschaft und Didaktik (S.144-167). Baltmannsweiler: Schneider-Verlag Hohengehren.

Thomé, G. (1992). Alphabetschrift und Schriftsystem. Über die Prinzipien der Orthographie aus schrifthistorischer Sicht. Zeitschrift für germanistische Linguistik, 20, 210 - 226.

Thomé, G. (2000). Silbe oder Morphem? Ein Beitrag aus der vergleichenden Schriftgeschichte zur Frage der Silbenorientierung unserer Schrift. In H. Balhorn, H. W. Giese \& C. Osburg (Hrsg.), Betrachtungen über Sprachbetrachtungen: Grammatik und Unterricht (S.106 -113). Seelze (Velber): Kallmeyer.

Thomé, G. (2019). Deutsche Orthographie. (2. verbesserte Aufl.). Oldenburg: isb Verlag.

Thomé, G. \& Eichler, W. (2008). Rechtschreiben Deutsch. In E. Klieme et al. (Hrsg.), Unterricht und Kompetenzerwerb in Deutsch und Englisch. Ergebnisse der DESI-Studie (S.104-111). Weinheim u.a.: Beltz,

Thomé, G. \& Thomé D. (2016). Deutsche Wörter nach Laut- und Schrifteinheiten gegliedert. Oldenburg: isb.

Thomé, G. \& Thomé, D. (2020). OLFA 3 - 9. Oldenburger Fehleranalyse für die Klassen 3 bis 9. Instrument und Handbuch. Oldenburg: isb.

Thomé, G., Corvacho del Toro, I. M. \& Thomé, D. (2011). Grundlagen qualitativer Fehleranalysen. In G. Schulte-Körne (Hrsg.), Legasthenie und Dyskalkulie Stärken erkennen - Stärken fördern (S. 43 - 49). Bochum: Winkler.
Thomé, G. Siekmann, K.; Thomé, D. (2011). Phonem-Graphem-Verhältnisse in der deutschen Orthographie: Ergebnisse einer neuen 100.000er Auszählung. In G. Schulte-Körne (Hrsg.), Legasthenie und Dyskalkulie. Stärken erkennen - Stärken fördern (S.51-64). Bochum: Winkler.

Treiman, R. \& Kessler, B. (2006). Spelling as Statistical Learning: Using Consonantal Context to Spell Vowels. Journal of Educational Psychology, 98 (3), $642-652$.

Trubetzkoy, N. S. (1989). Grundzüge der Phonologie. (7. Aufl.). Göttingen: Vandenhoeck \& Ruprecht.

Ulrich, T. (2018). Grammatikerwerb und grammatische Störungen im Kindesalter. Ergebnisse des Forschungsprojekts GED 4-9 und ihre Implikationen für sprachdiagnostische und -therapeutische Methoden. Habilitationsschrift, Universität zu Köln. Verfügbar unter https://kups.ub.uni-koeln.de/9011/

Volkmer, S., Schulte-Körne, G. \& Galuschka, K. (2019). Die Rolle der morphologischen Bewusstheit bei Lese- und Rechtschreibleistungen. Zeitschrift für Kinder- und Jugendpsychiatrie und Psychotherapie, 47 (4), 1 - 1.

\section{Historie}

Manuskript eingereicht: 24.04.2021

Manuskript angenommen: 20.05.2021

\section{Förderung}

Open-Access-Veröffentlichung ermöglicht durch die Universität Siegen.

\section{Danksagung}

Diese Studie fand im Rahmen des Projekts „Die Mittelstufe schreibt!", ein Kooperationsprojekt der Stiftung Polytechnische Gesellschaft Frankfurt am Main und der Goethe-Universität, statt. Unser Dank gilt der Stiftung Polytechnische Gesellschaft, den teilnehmenden Lehrerinnen und Lehrern, den Schülerinnen und Schülern, den studentischen Mitarbeiterinnen und Mitarbeitern sowie den Mitarbeiterinnen und Mitarbeitern der GoetheUniversität und der Stiftung Polytechnischen Gesellschaft, die in vielfältiger Weise am Projekt beteiligt waren.

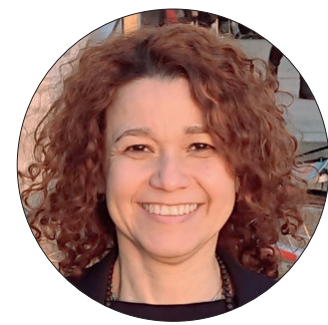

Dr. Irene Corvacho del Toro Akademische Oberrätin Germanistisches Seminar Universität Siegen Hölderlinstr. 3 57068 Siegen Deutschland corvacho.del.toro@germanistik. uni-siegen.de 